\title{
From RGB to Depth: Domain Transfer Network for Face Anti-Spoofing
}

\author{
Yahang Wang, Xiaoning Song*, Member, IEEE, Tianyang Xu, Zhenhua Feng, Member, IEEE \\ Xiao-Jun Wu, Member, IEEE
}

\begin{abstract}
With the rapid development in face recognition, most of the existing systems can perform very well in unconstrained scenarios. However, it is still a very challenging task to detect face spoofing attacks, thus face anti-spoofing has become one of the most important research topics in the community. Though various anti-spoofing models have been proposed, the generalisation capability of these models usually degrades for unseen attacks in the presence of challenging appearance variations, e.g., background, illumination, diverse spoofing materials and low image quality. To address this issue, we propose to use a Generative Adversarial Network (GAN) that transfers an input face image from the RGB domain to the depth domain. The generated depth clue enables biometric preservation against challenging appearance variations and diverse image qualities. To be more specific, the proposed method has two main stages. The first one is a GAN-based domain transfer module that converts an input image to its corresponding depth map. By design, a live face image should be transferred to a depth map whereas a spoofing face image should be transferred to a plain (black) image. The aim is to improve the discriminative capability of the proposed system. The second stage is a classification model that determines whether an input face image is live or spoofing. Benefit from the use of the GAN-based domain transfer module, the latent variables can effectively represent the depth information, complementarily enhancing the discrimination of the original RGB features. The experimental results obtained on several benchmarking datasets demonstrate the effectiveness of the proposed method, with superior performance over the stateof-the-art methods. The source code of the proposed method is publicly available at https://github.com/coderwangson/DFA.
\end{abstract}

Index Terms-Face anti-spoofing, generative adversarial network, domain transfer.

\section{INTRODUCTION}

$\mathbf{F}$ ACE recognition, as one of the most important research topics in computer vision and pattern recognition, has achieved great success in recent years [1], [2], [3], [4]. The technique has been widely used in a wide spectrum of practical

This work was supported in part by the National Key Research and Development Program of China (Grant No. 2017YFC1601800), the National Natural Science Foundation of China $(61876072,61902153)$, the China Postdoctoral Science Foundation (2018T110441) and the Six Talent Peaks Project of Jiangsu Province (XYDXX-012)

Yahang Wang, Xiaoning Song (corresponding author) and Xiao-Jun Wu are with the School of Artificial Intelligence and Computer Science, Jiangnan University, Wuxi 214122, China. (email: 6181914028@stu.jiangnan.edu.cn, x.song@jiangnan.edu.cn,wu_xiaojun@jiangnan.edu.cn)

Tianyang $\mathrm{Xu}$ is with the Centre for Vision, Speech and Signal Processing, University of Surrey, Guildford, GU2 7XH, UK. (e-mail: tianyang.xu@surrey.ac.uk)

Zhenhua Feng is with the Department of Computer Science and the Centre for Vision, Speech and Signal Processing, University of Surrey, Guildford, GU2 7XH, UK. (e-mail: z.feng@ surrey.ac.uk)

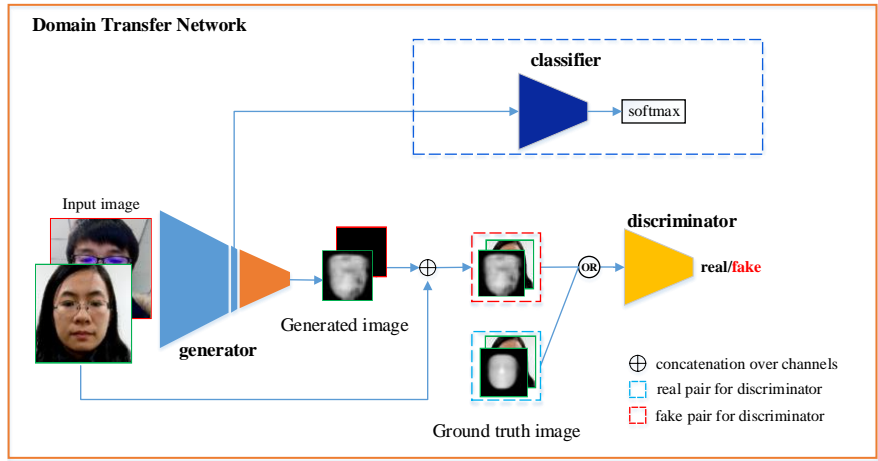

Fig. 1. The pipeline of the proposed Domain Transfer Network (DTN). Our DTN has three main parts: generator, discriminator and classifier. We can obtain better depth maps through adversarial learning of the generator and discriminator. Hence, the corresponding latent features can produce more robust classification results.

applications, such as CCTV surveillance, access control, financial security, human-computer interaction, etc. Therefore, it is crucial to guarantee the safety of a face recognition system. However, there is a massive human face data on the Internet, which can be misused to produce Presentation Attacks (PA) such as print attacks (printing a face on a paper) or video replay attacks (replaying a face video on a digital device). To mitigate this issue, Face Anti-Spoofing (FAS) plays a very important role for any facial image analysis system, aiming at determining whether an input face is live or spoofing.

To detect spoofing attacks, numerous face anti-spoofing methods have been proposed [5], [6], [7]. In the early stage, hand-crafted features, such as Local Binary Patterns (LBP) [8], Speeded Up Robust Features (SURF) [9], and Histogram of Oriented Gradients (HOG) [10], and shallow classifiers, such as support vector machine, are the most widely used techniques in FAS. However, the performance of hand-crafted features is always less effective in practical scenarios, including complex backgrounds and unpredictable spoofing types. In contrast to hand-crafted features, Convolutional Neural Networks (CNNs) have achieved impressive performance in many facial image analysis systems due to their powerful representation capability. In [11], [12], the community started exploring $\mathrm{CNN}$-based approaches for face anti-spoofing and received promising results. However, these methods may not able to generalise well for unseen samples due to the over-fitting problem. To learn more robust features for FAS, recent approaches use deep neural networks with auxiliary information, such as depth information, to support enhanced 


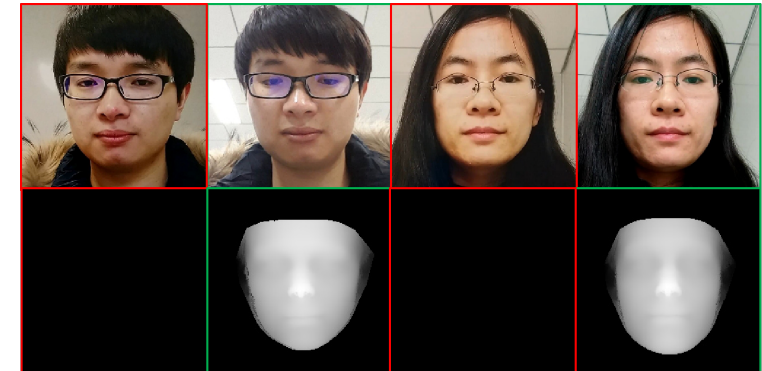

Fig. 2. A comparison of faces in the RGB domain (first row) and depth domain (second row) for face anti-spoofing: the first and third columns are spoofing faces, the second and fourth columns are live faces. By converting the images from RGB to depth, we can easily detect spoofing faces.

supervision [13], [14]. The performance improvement using depth information emphasises the necessity of exploring robust clues from auxiliary information rather than merely from the RGB domain, which inspires us to further exploit the potential of using depth information. To this aim, this paper analyses the effectiveness of depth information and aims to generate high-quality depth information for face anti-spoofing.

To intuitively compare RGB and depth images, we visualise several facial images in Fig. 2. We can see that the depth images are more distinctive than that of the RGB domain from the visual level. In Section III, we will further explore the effectiveness of depth information from the feature level. To highlight the significance of depth information, in this work, we present a novel method to learn a Domain Transfer Network (DTN) as illustrated in Fig. 1. In principle, we design a deep neural network that can generate high-quality depth images as a new domain. By design, the latent feature embedding of an input face image has both the information from the RGB and depth domains for face anti-spoofing. However, the existing publicly available face spoofing datasets, such as Replay-Attack [8] and CASIA-FASD [15], only have RGB faces, which impedes the use of depth information. Therefore, we generate depth faces of the training dataset in PRNet [16] as domain labels. After that, we use a Generative Adversarial Network (GAN) as the domain transfer model to convert RGB images to depth ones. There are two benefits of using the GAN model. First, we can leverage the capability of GAN to transform a face image from the RGB domain to the depth domain. Meanwhile, the latent variables of GAN contain the information extracted from the depth domain, providing discriminative features for face anti-spoofing. Second, since the discriminator of GAN is only needed during the network training stage, it can reduce the complexity of the network during inference and speed up the overall network, which is exactly what face spoofing detection needs.

To summarise, the main innovations of the proposed method include:

- In spite of fact that depth images have already been used in the existing face anti-spoofing systems, why the use of depth information has not been fully studied. To bridge this gap, we intuitively investigate the merits and disadvantages of the use of RGB images and depth images in face anti-spoofing from different dimensions.
- Due to the power generation capacity of GAN, we introduce GAN as a domain transfer module to generate more discriminative depth images for live and spoofing faces.

- We utilise the latent variables in the generator of the GAN module as the input of a spoofing detection classifier with binary supervision.

- Our proposed method make comprehensive comparisons and achieve state-of-the-art performance on widely used face anti-spoofing benchmarks with both intra- and crossdataset testing.

The rest of this paper is organised as follows. We first introduce the related work in Section II. Then we present the details of the proposed DTN method in Section III. The experimental results are reported and analysed in Section IV and the conclusion is drawn in Section V.

\section{RELATED WORK}

In this section, we review the relevant methods of face antispoofing and GAN. The existing methods of face anti-spoofing can be roughly divided into two categories: traditional methods and CNN-based methods.

\section{A. Traditional methods}

Traditional face anti-spoofing methods usually employ hand-crafted features, such as LBP [8], [17], HOG [10], SURF [9], IQA [18] and SIFT [19], equipped with shallow classifiers, e.g., SVM and LDA, to detect spoofing faces from live ones in a two-class classification manner. These approaches heavily rely on the assumption that the texture information of an RGB face image is valid to guide face antispoofing. However, since these manually designed features are sensitive to illumination variations, traditional methods often perform poorly in unconstrained scenarios. To address this issue, the use of different input domains, such as HSV and YCbCr spaces [20], [21], has been explored. In addition, temporal clues in sequential video frames also received wide attention in the area. For example, Pan et al. [22] tried to use eye blink and Kollreider et al. [23] applied the lip and mouth motion to detect face liveness. Since these methods exploit empirical knowledge, such as eye blink or lip motion, they are simple yet effective against printing attacks. But the above approaches have difficulties in detecting replay attacks. Similarly, to enhance the prior empirical knowledge, Komulainen et al. [24] concatenated the features of sequential frames to improve the performance. Additionally, some temporal features have also been studied, such as Haralick features [25], motion mag [26], and optical flow [27]. In general, there are certain limitations of traditional methods. First, they are not suitable for different types of attacks, such as printing and video replay attacks. Second, due to the diversity of datasets, traditional methods cannot generalise well in cross-database settings.

\section{B. CNN-based approaches}

In recent years, CNNs have demonstrated promising results in a wide range of computer vision and pattern recognition applications, including face anti-spoofing and other facial image 
analysis tasks [11], [3], [12], [28], [29], [30], [2]. However, most deep learning approaches regard face anti-spoofing as a simple binary classification problem using the softmax loss function for supervision. For example, Yang et al. [11] used $\mathrm{CNN}$ as a binary classifier that directly classifies live and spoofing faces. Similarly, [30] used the pre-trained VGG-face model as a feature extractor for classification. To emphasise the training of discriminative features, Feng et al. [31] used multi-view RGB faces as an input. In addition, [13] divided the original input image into small patches for network training, which achieved further performance boost. Nevertheless, there are some issues in training a deep neural network with manually designed binary supervision. For example, they cannot obtain robust spoofing patterns, lacking intuitive explanation or theoretical rationale.

To address the above issues, recent deep learning methods usually use auxiliary information to support additional discrimination rather than direct binary supervision. This auxiliary information is based on empirical knowledge, exploring the differences between live and spoofing faces. The most commonly used supervision is depth images. Atoum et al. [13] proposed to utilise depth maps as supervision to detect spoofing attacks. They proposed a two-stream CNN-based method for face anti-spoofing, by extracting the features of original face images and the corresponding depth maps. Liu et al. [14] enhanced this framework with rPPG signals from face videos. Besides, there are also approaches relying on temporal features, instead of spatial features. For instance, Xu et al. [32] introduced the CNN-LSTM structure that uses CNN to learn the spatial feature information of each frame and LSTM to learn the temporal information across frames. Similarly, the 3D CNN model [33] also considered a useful message from the temporal perspective. However, there are various types of spoofing attacks, which limits the generalisation capability of a face anti-spoofing system. To address this issue, more recent studies follow the domain generalisation framework [34], [35] to enhance the discriminative capacity of a face anti-spoofing model. Besides the focus on improving the model capacity, recent studies tried to transfer the two-class classification problem to a one-class task by reformulating the spoofing detection as an anomaly detection problem [36], [37], in which spoofing attacks are considered as outliers.

\section{Generative Adversarial Networks}

In recent years, GAN has become one of the most popular generative models in computer vision [38]. GAN can generate high-quality realistic images via the adversarial training process. For example, Cong et al. [39] proposed a dual encoderdecoder GAN that can generate realistic facial images with continuous pose variations. Song et al. [40] designed a selfgrowing and pruning GAN to improve the stability of network training and the quality of generated images. Additionally, GAN has been widely used for various image-to-image transfer tasks, such as style transfer [41], in-painting [42] and super resolution [43]. It has been proven that GAN can transfer images from one domain to another domain. But these approaches only adopt GAN for specific applications.
To address this issue, [44] developed a general framework for all the above tasks. In this paper, we propose to convert RGB face images to their depth maps, which can be considered as an image-to-image transfer problem.

\section{The PROPOSED FrameWORK}

\section{A. Analysis of RGB images for face anti-spoofing}

Most existing face anti-spoofing systems use RGB images for hand-crafted feature extraction, such as LBP, HOG, etc. Some methods also use CNN features and a fully connected layer for classification. Although the above paradigms have achieved promising results in face anti-spoofing, their common limitation is the poor generalisation capability. For face spoofing detection, it is difficult to find a common feature/pattern that can well represent spoofing clues due to the diversity and uncertainty of spoofing attacks. Though many solutions use deep neural networks for learning-based feature embedding, they all suffer from the over-fitting issue. These methods only perform well for the test samples with similar spoofing types of the training set, but cannot work well for unseen spoofing attacks.

The CNN features extracted from RGB images does not persistently valid for distinguishing live and spoofing faces. As we know, CNNs have been widely used for the tasks with pure textural information, such as image classification and face recognition. In Fig. 2, the first row shows some RGB images with the first and third ones as spoofing faces and the remaining ones as live faces. The second row shows the corresponding depth faces. For the first row, we would most likely misclassify them as the same category in spoofing detection. In contrast, we can easily identify the spoofing faces using the depth images in the second row. By training a CNN for face anti-spoofing with only RGB images, we use the objective function to force the network to memorise this information instead of learning. Therefore, it is challenging for an existing method to obtain discriminative face representations that can separate live and spoofing images using only RGB information.

To intuitively illustrate this issue at the feature level, we use Principal Component Analysis (PCA) to visualise CNN features in Fig. 4. First, we train two ResNet50 [45] models using the Replay-Attack dataset. The inputs of the first model, 'model-R', are RGB images. The inputs of the second model, 'model-D', are depth facial maps generated by PRNet. Second, we use the RGB data of Replay-Attack to test model-R. We use PCA to project the extracted CNN features of model-R into a three-dimensional space, as shown in Fig. 4-(a). We also visualise the CNN features extracted from the RGB images of CASIA-FASD by model-R in Fig. 4-(b). Similarly, we visualise the CNN features extracted from the depth images of the Replay-Attack and CASIA-FASD datasets by model-D in Fig. 4-(c) and Fig. 4-(d). According to Fig. 4-(a), we can see that the obtained features of live and spoofing faces can be well separated since the model was trained on the same dataset, i.e., the Replay-Attack dataset. However, for the CASIA-FASD test set, the CNN features of live and spoofing faces become harder to separate, as shown in Fig. 4-(b). This means that the CNN 


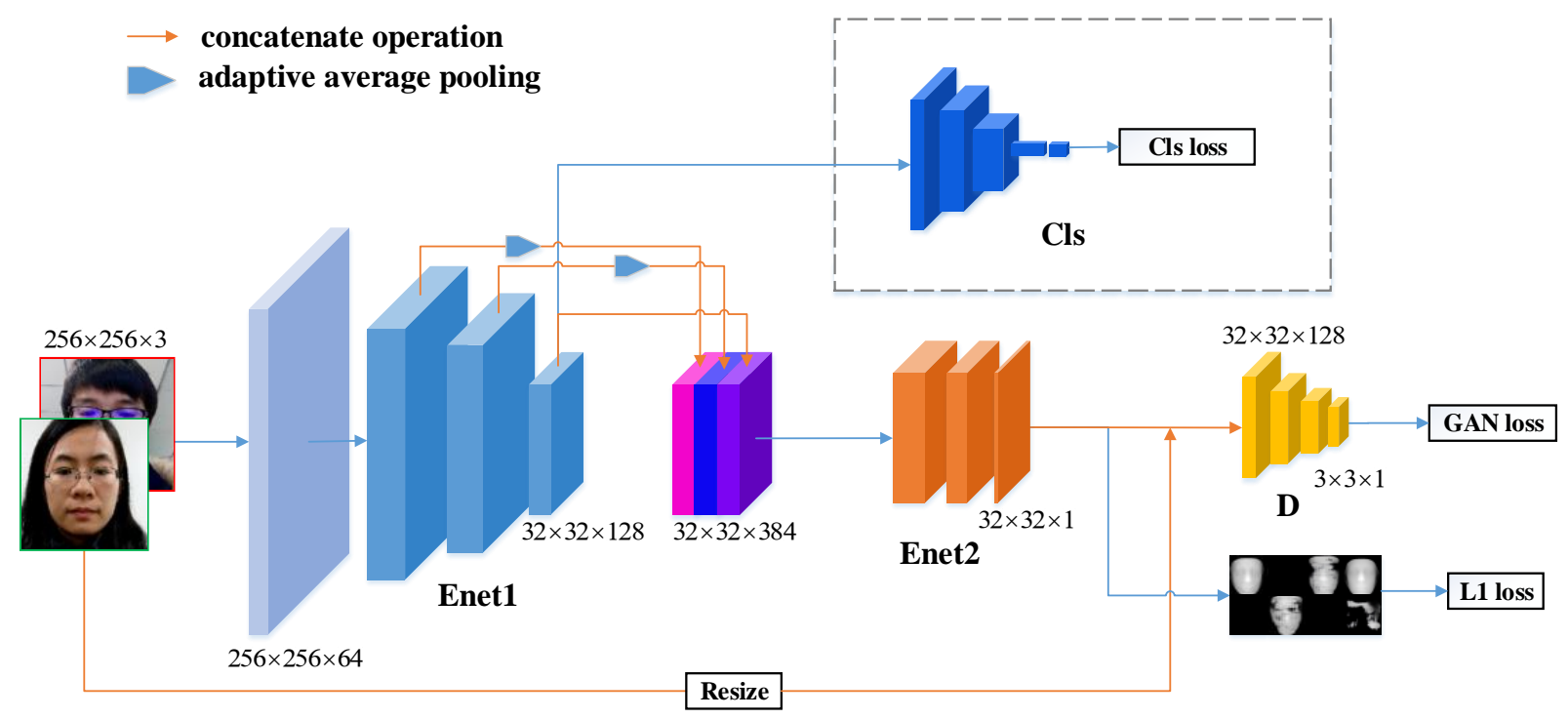

Fig. 3. The proposed Domain Transfer Network (DTN). The generator is composed of Enet 1 and Enet2, $D$ is the discriminator, $C l s$ is the classifier. Enet1 is divided into two branches for Cls and Enet2. With Enet2, we can generate the depth image for an input face.

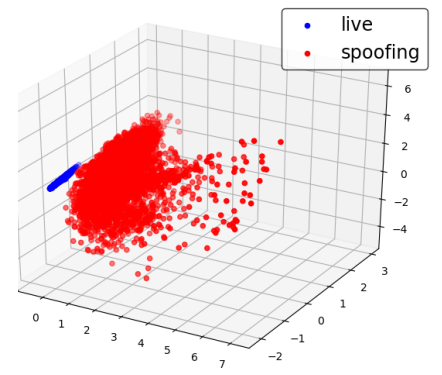

(a) RGB of Replay-Attack

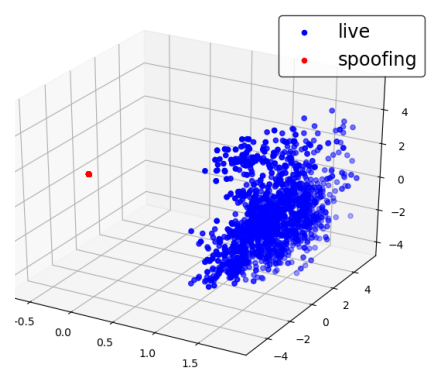

(c) Depth of Replay-Attack

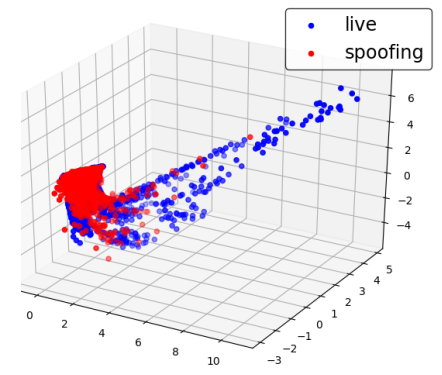

(b) RGB of CASIA-FASD

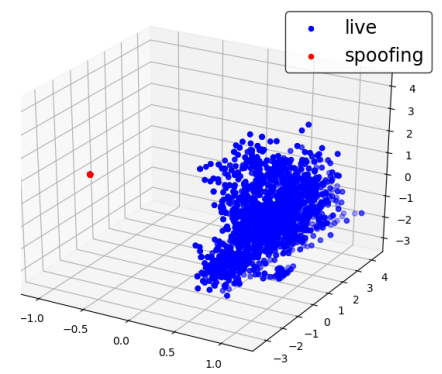

(d) Depth of CASIA-FASD

Fig. 4. Visualisation of CNN features extracted by ResNet50 in a projected 3D space using PCA. The ResNet50 model was trained on the Replay-Attack dataset. We compare the distributions of CNN features extracted from (a) the RGB images of the Replay-Attack test set; (b) the RGB images of the CASIA-FASD dataset; (c) the depth images of the Replay-Attack test set; and (d) the depth images of the CASIA-FASD dataset. Note that, we used PRNet to generate depth images for live faces.

model trained on Reply-Attacked is over-fitted thus it cannot generalise well for CASIA-FASD. Meanwhile, we can see that the distribution of live and spoofing samples in Fig. 4-(a) is different from that in Fig. 4-(b). This also explains why the model does not work well when the training and testing sets

are from different datasets. In contrast, for the visualisation results of the depth domain in Fig. 4-(c) and Fig. 4-(d), we can see that the obtained CNN features of live and spoofing faces can always be easily separated. Additionally, the distributions of live and spoofing faces are similar across different datasets. It should be noted that we do not have depth images for the Replay-Attack and CASIA-FASD datasets, so we simulate depth images for live faces with PRNet and use plain (black) images for spoofing faces. Therefore, all the spoofing samples are gathered together.

Through the above analysis, we can see that the depth image domain is more discriminative for face anti-spoofing. The CNN features extracted from depth images are more robust, thus a classifier can generalise well across datasets. However, the existing publicly available face spoofing datasets usually only have RGB faces, so it is impossible to directly use the information of the depth domain. To address this issue, we use GAN to generate depth images for live faces. GAN has been widely used for image generation and style transfer in computer vision. For example, the pix2pix method [44] was proposed to transfer images from one domain to another domain. To be more specific, we construct a GAN module to transfer faces from the RGB domain to the depth domain and exploit the generation capability of GAN to obtain reliable depth domain information.

\section{B. System overview}

To mitigate the aforementioned issue, we propose a novel face anti-spoofing framework that transfers RGB faces to their corresponding depth maps and performs live/spoofing face classification with latent variables, as depicted in Fig. 3. The proposed network consists of two deep networks. First, we develop a domain transfer network to convert RGB faces to depth maps. As discussed earlier, many existing studies use 
TABLE I

CONFIGURATION OF EACH CONVOLUTION BLOCK IN ENET1, WHERE $s$ AND $p$ REPRESENT STRIDE AND PADDING.

\begin{tabular}{lcc}
\hline Layer & Configuration & Output channel \\
\hline $\begin{array}{l}\text { Convolution } \\
\text { BatchNorm + ReLu }\end{array}$ & $3 \times 3, \mathrm{~s}=1, \mathrm{p}=1$ & 128 \\
$\begin{array}{l}\text { Convolution } \\
\text { BatchNorm + ReLu }\end{array}$ & $3 \times 3, \mathrm{~s}=1, \mathrm{p}=1$ & 196 \\
$\begin{array}{l}\text { Convolution } \\
\text { BatchNorm + ReLu }\end{array}$ & $3 \times 3, \mathrm{~s}=1, \mathrm{p}=1$ & 128 \\
Maxpool & $2 \times 2$ & \\
\hline
\end{tabular}

GAN for image generation and style transfer. Therefore, we follow the adversarial learning paradigm to obtain high-quality depth images with the generator and discriminator. Second, the proposed framework has a classifier to determine whether an input face image is live or spoofing. To train the proposed network in an end-to-end manner, we use the latent variables learned in the domain transfer stage as the input of the classier, as shown in Fig. 3.

1) Domain transfer: We design a GAN model for domain transfer from RGB to depth images. The proposed GAN model has a generator and a discriminator. To be more specific, our generator has two encoding parts, Enet 1 and Enet2. Enet 1 is designed for feature extraction, which contains multiple blocks of convolutional layers. The configuration of each convolutional block of Enet 1 is shown in Table I. Each convolutional layer is followed by one batch normalisation layer and one ReLu non-linear activation layer. The input image is $x \in \mathbb{R}^{256 \times 256}$ for the network. From Fig. 3 we can see that Enet 1 has two outputs. The first output is the latent variables extracted from the original RGB image. We will introduce more details of the latent variables in the next subsection. The second output is the response map that is concatenated by applying adaptive average pooling to the first two convolutional blocks. The aim is to fully utilise the information extracted from different layers. The second output will be used as the input of Enet2 that obtains the depth image for a given RGB face image. The forward pass of the generator can be formulated as below:

$$
\begin{aligned}
& z=\operatorname{Enet} 1(x), \\
& \hat{d}=\operatorname{Enet} 2\left(z^{\prime}\right),
\end{aligned}
$$

where $z$ represents the latent variables, $z^{\prime}$ represents the stacked features and $\hat{d}$ represents the generated depth image. In order to obtain high-quality depth images, we follow the GAN paradigm that uses a discriminator to classify real and fake depth images. The discriminator is designed as a Fully Convolutional Network (FCN) that contains multiple convolutional layers. Each convolutional layer is followed by one batch normalisation layer and one ReLu activation layer. During the training process, the depth image generator is trained to compete against the discriminator so as to gradually learn how to generate depth images from RGB faces. The objective of the proposed depth domain transfer network is:

$$
\mathcal{L}_{G A N}(G, D)=\mathbb{E}_{x, d}[\log D(x, d)]+\mathbb{E}_{x}[\log (1-D(x, \hat{d})],
$$

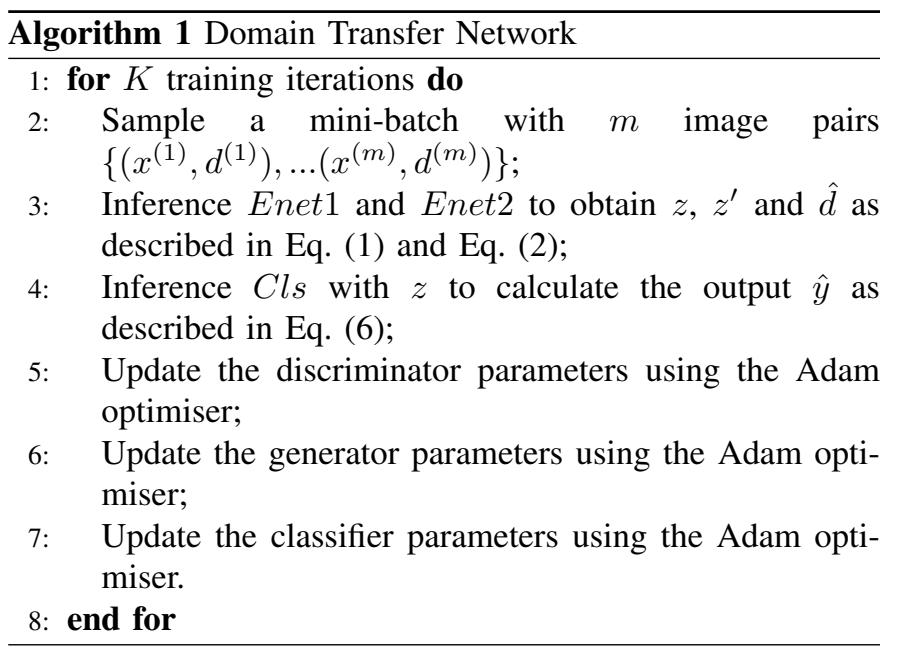

where $d$ is the ground truth depth image, $G$ and $D$ are the generator and discriminator. The training of GAN is a zerosum game and the stability of network training is crucial. Existing approaches usually use additional image reconstruction losses, such as L1 or L2 distance, to improve the quality of generated images as well as the stability of network training. With the reconstruction loss, the task of discriminator remains unchanged, but the generator is designed to not only fool the discriminator but also generate depth images that are close to the ground truth ones. In the proposed method, we use the L1 reconstruction loss:

$$
\mathcal{L}_{L_{1}}(G)=\mathbb{E}_{x, d}\left[\|d-\hat{d}\|_{1}\right]
$$

Last, we optimise the generator using the objective as follows:

$$
G^{*}=\arg \min _{G} \max _{D} \mathcal{L}_{G A N}(G, D)+\lambda \mathcal{L}_{L 1}(G),
$$

where $\lambda$ is a balancing parameter.

2) Classification: The classifier aims to decide whether the input face image is a live or spoofing face. As shown in Fig. 3, we utilise multiple convolutional layers, with one additional fully connected layer that converts the feature map into 0 or 1 for binary classification. As aforementioned, the input comes from one part of the proposed DTN branch. Specifically, it uses the feature maps generated by Enet 1 . Since the feature maps are generated during the domain transfer process, they can be regarded as features of the depth domain. As discussed earlier, compared with the information extracted from the RGB domain, the proposed method can gradually learn the shared features supervised by the depth domain transfer network. We optimise the classifier by minimising the cross-entropy loss:

$$
\mathcal{L}_{c}(C l s)=\frac{1}{N} \sum_{i}-\left[y_{i} \log \left(\hat{y}_{i}\right)+\left(1-y_{i}\right) \log \left(1-\hat{y}_{i}\right)\right]
$$

where $y$ is the binary label and $\hat{y}$ is the network prediction.

Last, we summarise the pipeline of the proposed DTN method in Algorithm 1. 


\section{EXPERIMENTS}

\section{A. Datasets and evaluation metrics}

1) Datasets: We evaluated the proposed method on four publicly available benchmarking datasets: CASIA-FASD [15], Replay-Attack [8], OULU-NPU [46] and MSU-MFSD [47].

CASIA-FASD has 600 videos of genuine and attack faces of 50 subjects. It was divided into the training and test sets of 20 and 30 subjects, respectively. The videos were captured by three types of cameras with diverse imaging qualities. CASIAFASD has three types of spoofing attacks, i.e., warped photo attack, cut photo attack and video attack. The benchmark has 7 testing scenarios, i.e., three fake face types, three imaging qualities and the whole data. In this paper, we use the whole data for evaluation.

Replay-Attack consists of 1200 video recordings of live and spoofing faces for 50 subjects. This database has two different illumination conditions (controlled and adverse) and two different support conditions (hand and fixed). For performance evaluation, the dataset was divided into three subsets: training (360 videos), development (306 videos) and testing (480 videos). The training and development sets have 300 attack videos and 60 real access videos respectively, whereas the testing set consists of 400 attacks and 80 real accesses. There are three types of spoofing attacks in Replay-Attack, i.e., print attack, mobile attack and high definition attack.

OULU-NPU contains 5940 video sequences of real accesses and attack attempts of 55 subjects (15 female and 40 male). This database was collected in three sessions with different illumination conditions, recorded using front-facing cameras of six different smartphones. Four kinds of attacks were designed, i.e., two print attack (Printer 1 and 2), two display devices (Display 1 and 2). Furthermore, the dataset was split into three disjoint subsets as training (20 users), development (15 users) and testing (20 users) sets.

MSU-MFSD consists of 280 video clips of photo and video attack attempts to 35 clients. Two types of cameras were used for data collection. Three types of spoof attacks were generated, using three different spoof medium to manifest the clients' biometrics: high-resolution replay video attacks using an iPad Air screen, mobile phone replay video attacks using an iPhone $5 \mathrm{~S}$ screen and printed photo attacks using an A3 paper with fully-occupied printed photo of a client.

2) Metrics: To make a fair comparison with existing studies, we follow the original evaluation metrics associated with each benchmarking dataset. For CASIA-FASD, we train and optimise model parameters using the training set and report the results in Equal Error Rate (EER) on the test set. We adopted the Half Total Error Rate (HTER) for Replay-Attack using the protocol defined in [8]. The HTER metric is obtained by calculating the mean of False Rejection Rate (FRR) and the False Acceptance Rate (FAR): HTER $=(F R R+F A R) / 2$. For the OULU-NPU dataset, we followed the standard ISO/IEC 30107-3 metrics [48], using APCER (Attack Presentation Classification Error Rate), BPCER (Bona Fide Presentation Classification Error Rate) and ACER (Average Classification Error Rate) that is the mean of APCER and BPCER: $A C E R=(A P C E R+B P C E R) / 2$.

\section{B. Implementation details}

The proposed method was implemented by using the PyTorch deep learning framework. The details of the proposed network are reported in Fig. 3 and Table I. The source code of the proposed method is publicly available at https://github. com/coderwangson/DFA. Inspired by DenseNet [49], we also used the lateral connection that concatenates the feature maps of three blocks. We used adaptive average pooling to normalise the three feature maps to the same spatial resolution. We fed the concatenated features to Enet 2 to generate depth facial images.

To be consistent with existing works, we performed frame sampling and face alignment for image pre-processing. As the evaluation benchmarks were recorded in videos, we first extracted the frames from each video. Subsequently, we detected faces using MTCNN [50] and generated depth faces using PRNet [16]. The size of each input image is $256 \times 256 \times 3$. We performed colour jitter, random horizontal flip and normalisation for data augmentation. For network training, we set the mini-batch size to 32 and the learning rate to $3 \mathrm{e}-5$. We used the Adam optimiser for network training [51]. The experiments were performed on an NVIDIA GTX 1080Ti card. The settings of different datasets are fixed for all the experiments.

\section{Experimental results}

1) Ablation study: In this section, we demonstrate the effectiveness of the proposed components by implementing three architectures on protocol 2 of OULU-NPU. First, we design a baseline network that consists of multiple convolutions with binary supervision, denoted by Model1. From Table II, we can see that Model1 achieves 5.12\% in terms of ACER. Second, we add an extra depth loss to Model1 as an extra supervision but without adversarial learning, which results in Model2. This verifies the effectiveness of the use of depth information by reducing the ACER to $3.57 \%$. However, due to the lack of an accurate depth image transfer model, effective depth information cannot be obtained. Last, we use GAN for image transfer to generate effective depth information through adversarial learning, which is denoted as Model3. We can see that Model3 achieves the best ACER that is $2.88 \%$, which demonstrates the effectiveness of our approach.

To further explore the effectiveness of the proposed method, we perform sensitivity analysis of the parameters for each component of the network. As shown in Fig. 3, the proposed network contains three losses. We report the results of the proposed method by assigning different weights to these losses in Table III. According to the table, we can see that the best result is achieved when set the weight of the classification loss to 1 , the weight of the $\mathrm{L} 1$ reconstruction loss to 100 , and the weight of the discriminator loss to 1 .

2) Closed-set evaluation: In this section, we first compare the proposed method with the state-of-the-art methods on several well-known benchmarking datasets, including CASIAFASD, Replay-Attack and OULU-NPU. We use the closed-set evaluation protocol, in which the training set and testing set are from the same dataset. 
TABLE II

A COMPARISON OF DIFFERENT MODELS ON PROTOCOL 2 OF OULU-NPU.

\begin{tabular}{cccc}
\hline Methods & APCER $(\%)$ & BPCER(\%) & ACER $(\%)$ \\
\hline Model1 & 5.47 & 4.78 & 5.12 \\
Model2 & 3.18 & 3.96 & 3.57 \\
Model3 & 3.84 & 2.11 & $\mathbf{2 . 8 8}$ \\
\hline
\end{tabular}

TABLE III

SENSITIVITY ANALYSIS OF THE WEIGHTS OF DIFFERENT LOSSES ON PROTOCOL 2 OF OULU-NPU.

\begin{tabular}{cccc}
\hline Cls & L1 & GAN & ACER(\%) \\
\hline 1 & 50 & 1 & 2.93 \\
1 & 100 & 1 & $\mathbf{2 . 8 8}$ \\
1 & 150 & 1 & 3.43 \\
\hline 5 & 100 & 1 & 3.38 \\
\hline 1 & 100 & 10 & 3.33 \\
\hline
\end{tabular}

TABLE IV

A COMPARISON WITH SOTA METHODS ON CASIA-FASD.

\begin{tabular}{lc}
\hline Method & EER(\%) \\
\hline DoG [15] & 17.00 \\
LBP [8] & 18.2 \\
Color texture [20] & 6.20 \\
CNN [11] & 4.64 \\
CNN+LSTM [32] & 5.17 \\
Patch and Depth [13] & 2.67 \\
FARCNN [52] & 2.35 \\
ML-DAN [53] & 3.7 \\
3D-CNN [54] & 1.4 \\
Attention [55] & 3.14 \\
\hline Our Method & $\mathbf{1 . 3 4}$ \\
\hline
\end{tabular}

TABLE V

A COMPARISON WITH SOTA METHODS ON REPLAY-ATTACK.

\begin{tabular}{lcc}
\hline Method & EER $(\%)$ & HTER(\%) \\
\hline LBP [8] & 13.9 & 13.8 \\
IQA [56] & - & 15.2 \\
CNN [11] & 4.64 & - \\
Color texture [20] & 0.4 & 2.9 \\
Patch and Depth [13] & 0.79 & 0.72 \\
FARCNN [52] & 0.06 & 0.18 \\
ML-DAN [53] & 0.3 & 0.6 \\
3D-CNN [54] & 0.3 & 1.2 \\
Attention [55] & 0.13 & 0.25 \\
\hline Our Method & $\mathbf{0 . 0 6}$ & $\mathbf{0 . 0 2}$ \\
\hline
\end{tabular}

The results obtained on CASIA-FASD are reported in IV using the EER metric. For the Replay-Attack dataset, we follow the protocols [8] and report the EER and HTER results of different methods in Table V. We compare the proposed method with different methods, including both handcrafted feature based methods and deep learning based methods. According to the results in Table IV and Table V, we can see that our method beats all the existing face anti-spoofing methods. Although some methods [52], [55] performs similar to the proposed method in the closed-set evaluation, we consider the information of the generated depth maps by GAN for domain transfer. Therefore, the proposed method achieves more significant superiority over these approaches in the following open-set evaluation settings.

OULU-NPU has four protocols for the evaluation of the performance of a face presentation attack detection method. Protocol 1 is designed to evaluate the generalisation capa-
TABLE VI

A COMPARISON WITH SOTA METHODS ON OULU-NPU.

\begin{tabular}{clccc}
\hline Proc. & Method & APCER(\%) & BPCER(\%) & ACER(\%) \\
\hline \multirow{4}{*}{1} & CPqD [57] & 2.9 & 10.8 & 6.9 \\
& FaceDs [58] & 1.2 & 1.7 & 1.5 \\
& STASN [59] & 1.2 & 2.5 & 1.9 \\
& STDN [60] & 0.8 & 1.3 & 1.1 \\
& SGTD [61] & 2.0 & 0 & 1 \\
\cline { 2 - 5 } & Our Method & 0.78 & 1.06 & $\mathbf{0 . 9 2}$ \\
\hline \multirow{4}{*}{2} & CPqD [57] & 14.7 & 3.6 & 9.2 \\
& FaceDs [58] & 4.2 & 4.4 & 4.3 \\
& STASN [59] & 4.2 & 0.3 & 2.2 \\
& STDN [60] & 2.3 & 1.6 & $\mathbf{1 . 9}$ \\
& SGTD [61] & 2.5 & 1.3 & $\mathbf{1 . 9}$ \\
\cline { 2 - 5 } & Our Method & 3.84 & 2.11 & 2.88 \\
\hline \multirow{4}{*}{3} & CPqD [57] & $6.8 \pm 5.6$ & $8.1 \pm 6.4$ & $7.4 \pm 3.3$ \\
& FaceDs [58] & $4.0 \pm 1.8$ & $3.8 \pm 1.2$ & $3.6 \pm 1.6$ \\
& STASN [59] & $4.7 \pm 3.9$ & $0.9 \pm 1.2$ & $2.8 \pm 1.6$ \\
& STDN [60] & $1.6 \pm 1.6$ & $4.0 \pm 5.4$ & $2.8 \pm 3.3$ \\
& SGTD [61] & $3.2 \pm 2.0$ & $2.2 \pm 1.4$ & $\mathbf{2 . 7 \pm \mathbf { 0 . 6 }}$ \\
\cline { 2 - 5 } & Our Method & $1.9 \pm 1.6$ & $3.8 \pm 6.4$ & $2.8 \pm 2.7$ \\
\hline \multirow{4}{*}{4} & CPqD [57] & $32.5 \pm 37.5$ & $11.7 \pm 12.1$ & $22.1 \pm 20.8$ \\
& FaceDs [58] & $1.2 \pm 6.3$ & $6.1 \pm 5.1$ & $5.6 \pm 5.7$ \\
& STASN [59] & $6.7 \pm 10.6$ & $8.3 \pm 8.4$ & $7.5 \pm 4.7$ \\
& STDN [60] & $2.3 \pm 3.6$ & $5.2 \pm 5.4$ & $3.8 \pm 4.2$ \\
& SGTD [61] & $6.7 \pm 7.5$ & $3.3 \pm 4.1$ & $5.0 \pm 2.2$ \\
\hline & Our Method & $4.0 \pm 4.1$ & $3.0 \pm 4.9$ & $\mathbf{3 . 5} \pm 2.4$
\end{tabular}

bility of a method for unseen illumination and background variations. Protocol 2 is designed to evaluate the generalisation capability of a method for unseen attack medium, such as unseen printers or displays. Protocol 3 evaluates the effect of image capturing device (camera) variations using a leave-one-camera-out protocol. Protocol 4 investigates all the above factors. Compared with the CASIA-FASD and ReplayAttack benchmarks, OULU-NPU has higher requirements on the generalisation capability of a face anti-spoofing model. Table VI reports the results of the proposed method and a comparison with the state-of-the-art approaches [57], [58], [59], [60], [61] on different protocols. We can see that the proposed method achieves the best results in protocol 1 and protocol 4, while obtains competitive results on protocol 2 and protocol 3. For protocol 3, the proposed method performs only slightly worse than SGTD. As protocol 4 investigates all the challenging factors of the OULU-NPU dataset, the result verifies the generalisation capability and effectiveness of the proposed method.

3) Open-set evaluation: Since different datasets were captured with different devices and spoof methods under different environments, it is interesting to evaluate the performance of a model in an open-set setting. Many recent studies use the open-set evaluation protocol, in which a model is trained and tested on different datasets to verify the generalisation capability of the model. We also perform open-set evaluation on CASIA-FASD and Replay-Attack. To be more specific, we use two protocols. The first one is to train a model on CASIA-FASD and test it on Replay-Attack. For the second protocol, we train a model on Replay-Attack and test it on CASIA-FASD. Because CASIA-FASD and Replay-Attack are different in image quality, image capturing method and environment, the two protocols can prove the generalisation ability of an algorithm. We compare the proposed method with 
TABLE VII

OPEN-SET EVALUATION RESULTS IN TERMS OF HTER (\%) AND AUC (\%) ON FOUR DATASETS.

\begin{tabular}{lcccccccc}
\hline \multirow{2}{*}{ Method } & \multicolumn{2}{c}{ O\&C\&I to M } & \multicolumn{2}{c}{ O\&M\&I to C } & \multicolumn{2}{c}{ O\&C\&M to I } & \multicolumn{2}{c}{ I\&C\&M to O } \\
\cline { 2 - 9 } & HTER(\%) & AUC $(\%)$ & HTER $(\%)$ & AUC $(\%)$ & HTER(\%) & AUC $(\%)$ & HTER(\%) & AUC $(\%)$ \\
\hline Binary CNN [11] & 29.25 & 82.87 & 34.88 & 71.94 & 34.47 & 65.88 & 29.61 & 77.54 \\
LBP TOP [17] & 36.9 & 70.80 & 42.6 & 61.05 & 49.45 & 49.54 & 53.15 & 44.09 \\
Auxiliary(Depth Only) [14] & 22.72 & 85.88 & 33.52 & 73.15 & 29.14 & 71.69 & 30.17 & 77.61 \\
MADDG [62] & 17.69 & $\mathbf{8 8 . 0 6}$ & 24.5 & 84.51 & 22.19 & 84.99 & 27.98 & 80.02 \\
DR-UDA [63] & $\mathbf{1 6 . 1}$ & - & 22.2 & - & 22.7 & - & 24.7 & - \\
\hline Our Method & 19.40 & 86.87 & $\mathbf{2 2 . 0 3}$ & $\mathbf{8 7 . 7 1}$ & $\mathbf{2 1 . 4 3}$ & $\mathbf{8 8 . 8 1}$ & $\mathbf{1 8 . 2 6}$ & $\mathbf{8 9 . 4 0}$ \\
\hline
\end{tabular}

TABLE VIII

OPEN-SET EVALUATION RESULTS IN TERMS OF HTER (\%) ON THE CASIA-FASD AND REPLAY-ATTACK DATASETS.

\begin{tabular}{|c|c|c|c|c|}
\hline Method & $\begin{array}{l}\text { Train } \\
\text { CASIA- } \\
\text { FASD }\end{array}$ & $\begin{array}{l}\text { Test } \\
\text { Replay- } \\
\text { Attack }\end{array}$ & $\begin{array}{l}\text { Train } \\
\text { Replay- } \\
\text { Attack }\end{array}$ & $\begin{array}{l}\text { Test } \\
\text { CASIA- } \\
\text { FASD }\end{array}$ \\
\hline LBP [8] & \multicolumn{2}{|c|}{55.9} & \multicolumn{2}{|c|}{47.9} \\
\hline CNN [11] & \multicolumn{2}{|c|}{48.5} & \multicolumn{2}{|c|}{39.6} \\
\hline Color texture [20] & \multicolumn{2}{|c|}{47.0} & \multicolumn{2}{|c|}{39.6} \\
\hline LBP-TOP [17] & \multicolumn{2}{|c|}{49.7} & \multicolumn{2}{|c|}{60.6} \\
\hline Deep-Learning [64] & \multicolumn{2}{|c|}{48.2} & \multicolumn{2}{|c|}{45.4} \\
\hline Depth + rPPG [14] & \multicolumn{2}{|c|}{27.9} & \multicolumn{2}{|c|}{28.4} \\
\hline FARCNN [52] & \multirow{2}{*}{\multicolumn{2}{|c|}{26.0}} & \multicolumn{2}{|c|}{29.4} \\
\hline STASN [59] & & & \multicolumn{2}{|c|}{30.9} \\
\hline GFA-CNN [65] & \multicolumn{2}{|c|}{21.4} & \multicolumn{2}{|c|}{34.4} \\
\hline Attention [55] & \multicolumn{2}{|c|}{30.0} & \multirow{2}{*}{\multicolumn{2}{|c|}{$\begin{array}{r}33.4 \\
208\end{array}$}} \\
\hline Our Method & \multicolumn{2}{|c|}{16.64} & & \\
\hline
\end{tabular}

other approaches in Table VIII. According to the results, the proposed method achieves the best performance in terms of HTER, $16.64 \%$ and $22.98 \%$, on both protocols, outperforming all the other approaches.

To further verify the effectiveness of the proposed approach in more complex scenarios, we follow the same open-set evaluation protocol defined in [63]. This protocol uses four datasets: OULU-NPU (denoted as O), CASLA-FASD (denoted as C), Replay-Attack (denoted as I), and MSU-MFSD (denoted as M). Specifically, we randomly select one database as the test set and the remaining three as the training set, resulting in four settings: $O \& C \& I$ to $M, O \& M \& I$ to $C, O \& C \& M$ to $\mathrm{I}$, and I\&C\&M to O. The results are reported in Table VII. Again, the proposed method achieves the best results on most of the four open-set settings as compared with the state-of-theart approaches. The notable performance of our method on the above open-set evaluations further verifies the generalisation capability and superiority of the proposed method.

\section{Visualisation and analysis}

As aforementioned, the use of RGB faces is difficult to distinguish live and spoofing faces. To further verify the effectiveness of our method, we first visualise the generated depth faces in Fig. 5. Fig. 5-(a) shows the mean and standard deviation of the generated depth faces of all the test samples in OULU-NPU Protocol 2. In this figure, we compare the proposed DTN method (model3) with a model that does not have the adversarial training module (model2). We can see that both model 2 and model 3 have similar results in terms of mean and standard deviation. It is very difficulty to identify the advantages of the proposed method when averaging across

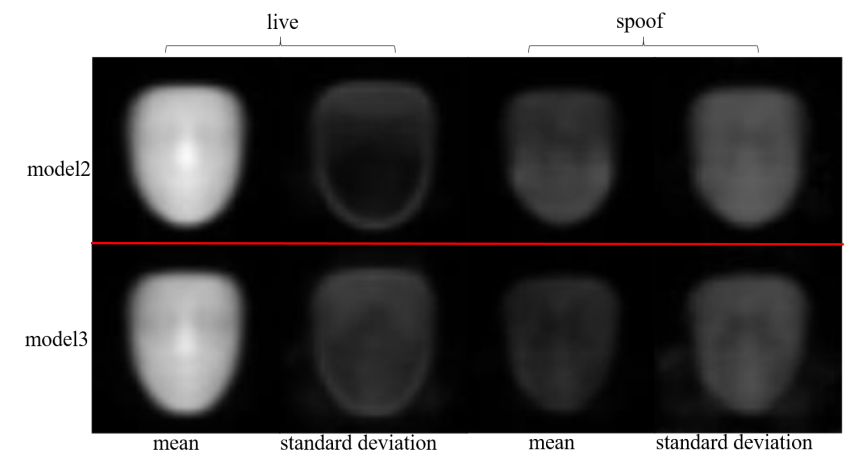

(a) The mean and standard deviation of the generated depth images by model 2 and model 3 .

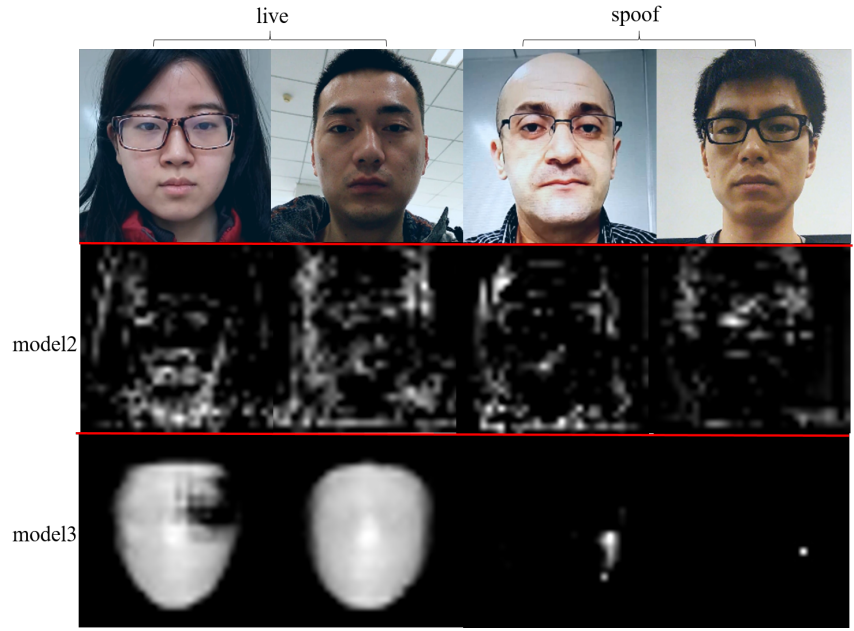

(b) The generated depth images of hard test samples in OULU-NPU.

Fig. 5. A comparison of the depth images generated by the proposed DTN method (model3) and those generated without adversarial training (model2): (a) the mean and standard deviation of the depth images obtained from the test samples; (b) the results of some hard test samples.

the whole dataset. However, we can see a clear difference between the two models in terms of the qualify of a generated depth image if we select some hard samples in OULU-NPU, as shown in Fig. 5-(b). We can see that our DTN can successfully transfer live RGB faces to their depth maps. In contrast, for spoofing faces, DTN tends to generate plain images only. Although it is difficult to distinguish live and spoofing faces in the original RGB domain, the differences between them are very clear as shown in the third row of the figure. By comparing with Fig. 2, we can see that our results are solid. We also provide the depth maps generated without the GAN 

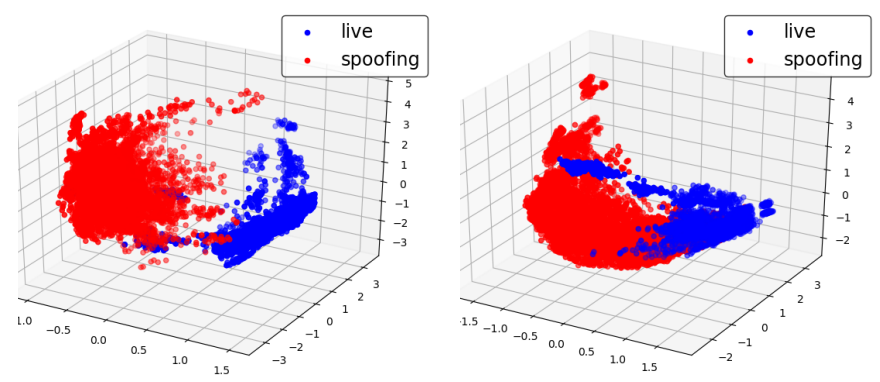

Fig. 6. Visualisation of the CNN features extracted by the proposed method for classification by projecting the $\mathrm{CNN}$ features into a 3D PCA subspace. The model is trained on the CASIA-FASD dataset. The left sub-figure shows the distribution of the CASIA-FASD test set and the right one shows the distribution of the Replay-Attack test set.

loss in the second row. Compared with the results output by our method, the quality of the depth maps generated without DTN are much worse, which further indicates the effectiveness of the proposed method.

To verify the discrimination of the extracted CNN features for face anti-spoofing, we use PCA to project the CNN features used for the classifier into a 3D subspace, as shown in Fig. 6. In the left sub-figure, we plot the feature distributions of the live and spoofing faces of the CASIA-FASD test set, using the model trained on the CASIA-FASD training set. For the right sub-figure, we plot the feature distributions of the live and spoofing faces of the Replay-Attack test set, using the model trained on the CASIA-FASD training set as well. We can see that the distributions of live and spoofing faces are very similar between the two sub-figures. Although the distributions in the right sub-figure are slightly shifted from the left ones, we can still easily distinguish real and spoofing faces. The shift is probably caused by the facial appearance variations in illumination and image resolution. As compared with Fig. 4(a) and Fig. 4-(b), the proposed method has improved the discrimination of CNN features significantly. The proposed DTN architecture converts RGB faces to the depth domain, which leads to similar feature distributions of real and spoofing faces among different datasets thus improves the generalisation capability of the proposed method.

To intuitively understand how the proposed method makes a decision, we also visualise the class activation maps by using the Gradient-weighted Class Activation Mapping (GradCAM) [66] technique. Grad-CAM uses the gradients of any target concept, flowing into the final convolutional layer to produce a coarse localisation map that highlights the important areas in the image for predicting the concept. As shown in Fig. 7, we visualise the class activation maps for some test examples. The Grad-CAM maps in the first and second rows are generated from live and spoofing faces, respectively. For a live face in the first row, the face region is focused because most of the depth information comes from the face region. In contrast, for a spoofing face in the second row, the attention distribution over the image is random as a spoofing face does not contain depth information. It is worth noting that our classification network is very shallow, but still captures the main area for attention. This further proves the importance of

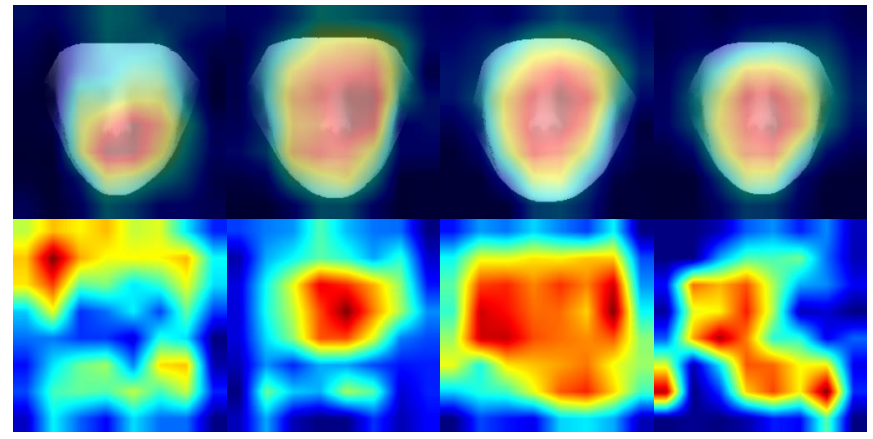

Fig. 7. Visualisation of the Grad-CAM attention maps of different test sample. The attention is focused on the faces for the live faces in the first row, while the attention is random for spoofing faces in the second row.

the use of depth information and our domain transfer network for face anti-spoofing.

\section{CONClusion}

In this paper, we proposed a novel face anti-spoofing framework, namely Domain Transfer Network (DTN), that effectively distinguishes live and spoofing faces. The proposed DTN method converts RGB faces to the depth domain for discriminative feature learning. Specifically, the proposed approach uses GAN for image domain transfer and employs CNN to extract latent depth information. By converting the original RGB face images to the depth domain, it enables the proposed method to alleviate the difficulties caused by appearance variations in lighting, image quality, image capturing device, etc. We evaluated the proposed method on several well-known benchmarking datasets using both closedset and open-set settings, with a comparison of the stateof-the-art approaches. The experimental results demonstrate the merits and superiority of the proposed method. However, the proposed method focuses on still images, ignoring the temporal information for face anti-spoofing, which may not able to work well for 3D spoofing attaches such as silicone masks. In our future work, we will combine some other solutions [67] to address this issue.

\section{REFERENCES}

[1] Yaniv Taigman, Ming Yang, Marc'Aurelio Ranzato, and Lior Wolf. Deepface: Closing the gap to human-level performance in face verification. In Proceedings of the IEEE conference on computer vision and pattern recognition, pages 1701-1708, 2014.

[2] Ali Akbari, Muhammad Awais, Zhenhua Feng, Ammarah Farooq, and Josef Kittler. Distribution cognisant loss for cross-database facial age estimation with sensitivity analysis. IEEE Transactions on Pattern Analysis and Machine Intelligence, 2020.

[3] Syed Safwan Khalid, Muhammad Awais, Zhen-Hua Feng, Chi-Ho Chan, Ammarah Farooq, Ali Akbari, and Josef Kittler. Resolution invariant face recognition using a distillation approach. IEEE Transactions on Biometrics, Behavior, and Identity Science, 2(4):410-420, 2020.

[4] Changbin Shao, Jing Huo, Lei Qi, Zhen-Hua Feng, Wenbin Li, Chuanqi Dong, and Yang Gao. Biased feature learning for occlusion invariant face recognition. In International Joint Conference on Artifical Intelligence, pages 666-672, 2020

[5] Javier Hernandez-Ortega, Julian Fierrez, Aythami Morales, and Javier Galbally. Introduction to face presentation attack detection. In Handbook of Biometric Anti-Spoofing, pages 187-206. Springer, 2019. 
[6] Abdenour Hadid, Nicholas Evans, Sébastien Marcel, and Julian Fierrez. Biometrics systems under spoofing attack: an evaluation methodology and lessons learned. IEEE Signal Processing Magazine, 32(5):20-30, 2015.

[7] Javier Galbally, Sébastien Marcel, and Julian Fierrez. Biometric antispoofing methods: A survey in face recognition. IEEE Access, 2:1530$1552,2014$.

[8] I. Chingovska, A. Anjos, and S. Marcel. On the effectiveness of local binary patterns in face anti-spoofing. In 2012 BIOSIG - Proceedings of the International Conference of Biometrics Special Interest Group (BIOSIG), pages 1-7, 2012.

[9] Z. Boulkenafet, J. Komulainen, and A. Hadid. Face antispoofing using speeded-up robust features and fisher vector encoding. IEEE Signal Processing Letters, 24(2):141-145, 2017.

[10] N. Dalal and B. Triggs. Histograms of oriented gradients for human detection. In 2005 IEEE Computer Society Conference on Computer Vision and Pattern Recognition (CVPR), volume 1, pages 886-893, 2005.

[11] J. Yang, Z. Lei, and S. Li. Learn Convolutional Neural Network for Face Anti-Spoofing. arXiv e-prints arXiv:1408.5601, 2014.

[12] L. Li, X. Feng, Z. Boulkenafet, Z. Xia, M. Li, and A. Hadid. An original face anti-spoofing approach using partial convolutional neural network. In 2016 Sixth International Conference on Image Processing Theory, Tools and Applications (IPTA), pages 1-6, 2016.

[13] Y. Atoum, Y. Liu, A. Jourabloo, and X. Liu. Face anti-spoofing using patch and depth-based cnns. In 2017 IEEE International Joint Conference on Biometrics (IJCB), pages 319-328, 2017.

[14] Y. Liu, A. Jourabloo, and X. Liu. Learning deep models for face antispoofing: Binary or auxiliary supervision. In 2018 IEEE Conference on Computer Vision and Pattern Recognition (CVPR), pages 389-398, 2018.

[15] Z. Zhang, J. Yan, S. Liu, Z. Lei, D. Yi, and S. Z. Li. A face antispoofing database with diverse attacks. In 2012 International Conference on Biometrics (ICB), pages 26-31, 2012.

[16] Y. Feng, F. Wu, X. Shao, Y. Wang, and X. Zhou. Joint 3d face reconstruction and dense alignment with position map regression network. In Computer Vision - ECCV 2018, pages 557-574. Springer International Publishing, 2018.

[17] T. Pereira, J. Komulainen, A. Anjos, J. Martino, A. Hadid, M. Pietikäinen, and S. Marcel. Face liveness detection using dynamic texture. EURASIP Journal on Image and Video Processing, 2014(1):2, 2014.

[18] Javier Galbally, Sébastien Marcel, and Julian Fierrez. Image quality assessment for fake biometric detection: Application to iris, fingerprint, and face recognition. IEEE transactions on image processing, 23(2):710-724, 2013.

[19] K. Patel, H. Han, and A. K. Jain. Secure face unlock: Spoof detection on smartphones. IEEE Transactions on Information Forensics and Security, 11(10):2268-2283, 2016

[20] Z. Boulkenafet, J. Komulainen, and A. Hadid. Face anti-spoofing based on color texture analysis. In 2015 IEEE International Conference on Image Processing (ICIP), pages 2636-2640, 2015.

[21] Z. Boulkenafet, J. Komulainen, and A. Hadid. Face spoofing detection using colour texture analysis. IEEE Transactions on Information Forensics and Security, 11(8):1818-1830, 2016.

[22] G. Pan, L. Sun, Z. Wu, and S. Lao. Eyeblink-based anti-spoofing in face recognition from a generic webcamera. In 2007 IEEE International Conference on Computer Vision (ICCV), pages 1-8, 2007.

[23] K. Kollreider, H. Fronthaler, M. I. Faraj, and J. Bigun. Real-time face detection and motion analysis with application in "liveness" assessment. IEEE Transactions on Information Forensics and Security, 2(3):548558, 2007.

[24] J. Komulainen, A. Hadid, M. Pietikäinen, A. Anjos, and S. Marcel. Complementary countermeasures for detecting scenic face spoofing attacks. In 2013 International Conference on Biometrics (ICB), pages $1-7,2013$.

[25] A. Agarwal, R. Singh, and M. Vatsa. Face anti-spoofing using haralick features. In 2016 IEEE 8th International Conference on Biometrics Theory, Applications and Systems (BTAS), pages 1-6, 2016.

[26] T. A. Siddiqui, S. Bharadwaj, T. I. Dhamecha, A. Agarwal, M. Vatsa, R. Singh, and N. Ratha. Face anti-spoofing with multifeature videolet aggregation. In 2016 23rd International Conference on Pattern Recognition (ICPR), pages 1035-1040, 2016.

[27] W. Bao, H. Li, N. Li, and W. Jiang. A liveness detection method for face recognition based on optical flow field. In 2009 International Conference on Image Analysis and Signal Processing, pages 233-236, 2009.
[28] O. Lucena, A. Junior, V. Moia, R. Souza, E. Valle, and R. Lotufo Transfer learning using convolutional neural networks for face antispoofing. In Image Analysis and Recognition, pages 27-34. Springer International Publishing, 2017.

[29] Zhen-Hua Feng, Josef Kittler, Muhammad Awais, and Xiao-Jun Wu. Rectified wing loss for efficient and robust facial landmark localisation with convolutional neural networks. International Journal of Computer Vision, pages 1-20, 2019

[30] K. Patel, H. Han, and A. Jain. Cross-database face antispoofing with robust feature representation. In Chinese Conference on Biometric Recognition, pages 611-619. Springer International Publishing, 2016.

[31] L. Feng, L. Po, Y. Li, X. Xu, F. Yuan, T. Cheung, and K. Cheung. Integration of image quality and motion cues for face anti-spoofing: A neural network approach. Journal of Visual Communication and Image Representation, 38:451-460, 2016.

[32] Z. Xu, S. Li, and W. Deng. Learning temporal features using lstm-cnn architecture for face anti-spoofing. In $20153 \mathrm{rd}$ IAPR Asian Conference on Pattern Recognition (ACPR), pages 141-145, 2015.

[33] J. Gan, S. Li, Y. Zhai, and C. Liu. 3d convolutional neural network based on face anti-spoofing. In 2017 2nd International Conference on Multimedia and Image Processing (ICMIP), pages 1-5, 2017.

[34] Y. Jia, J. Zhang, S. Shan, and X. Chen. Single-side domain generalization for face anti-spoofing. arXiv preprint arXiv:2004.14043, 2020.

[35] X. Tu, H. Zhang, M. Xie, Y. Luo, Y. Zhang, and Z. Ma. Deep transfer across domains for face antispoofing. Journal of Electronic Imaging, 28(4):043001, 2019.

[36] D. Pérez-Cabo, D. Jiménez-Cabello, A. Costa-Pazo, and R. LópezSastre. Deep anomaly detection for generalized face anti-spoofing. In 2019 IEEE Conference on Computer Vision and Pattern Recognition (CVPR) Workshops, 2019.

[37] S. Fatemifar, S. R. Arashloo, M. Awais, and J. Kittler. Spoofing attack detection by anomaly detection. In ICASSP 2019 - 2019 IEEE International Conference on Acoustics, Speech and Signal Processing (ICASSP), pages 8464-8468, 2019.

[38] I. Goodfellow, J. Pouget-Abadie, M. Mirza, B. Xu, D. Warde-Farley, S. Ozair, A. Courville, and Y. Bengio. Generative adversarial nets. In Advances in Neural Information Processing Systems 27, pages 26722680. Curran Associates, Inc., 2014

[39] Cong Hu, Zhenhua Feng, Xiaojun Wu, and Josef Kittler. Dual encoderdecoder based generative adversarial networks for disentangled facial representation learning. IEEE Access, 8:130159-130171, 2020.

[40] Xiaoning Song, Yao Chen, Zhen-Hua Feng, Guosheng Hu, Dong-Jun $\mathrm{Yu}$, and Xiao-Jun Wu. SP-GAN: Self-growing and pruning generative adversarial networks. IEEE Transactions on Neural Networks and Learning Systems, 32(6):2458-2469, 2020.

[41] C. Li and M. Wand. Precomputed real-time texture synthesis with markovian generative adversarial networks. In Computer Vision - ECCV 2016, pages 702-716. Springer International Publishing, 2016.

[42] D. Pathak, P. Krähenbühl, J. Donahue, T. Darrell, and A. A. Efros. Context encoders: Feature learning by inpainting. In 2016 IEEE Conference on Computer Vision and Pattern Recognition (CVPR), pages 2536-2544, 2016.

[43] C. Ledig, L. Theis, F. Huszár, J. Caballero, A. Cunningham, A. Acosta, A. Aitken, A. Tejani, J. Totz, Z. Wang, and W. Shi. Photo-realistic single image super-resolution using a generative adversarial network. In 2017 IEEE Conference on Computer Vision and Pattern Recognition (CVPR), pages $105-114,2017$

[44] P. Isola, J. Zhu, T. Zhou, and A. A. Efros. Image-to-image translation with conditional adversarial networks. In 2017 IEEE Conference on Computer Vision and Pattern Recognition (CVPR), pages 5967-5976, 2017.

[45] K. He, X. Zhang, S. Ren, and J. Sun. Deep residual learning for image recognition. In 2016 IEEE Conference on Computer Vision and Pattern Recognition (CVPR), June 2016.

[46] Z. Boulkenafet, J. Komulainen, L. Li, X. Feng, and A. Hadid. Oulu-npu: A mobile face presentation attack database with real-world variations. In 2017 12th IEEE International Conference on Automatic Face Gesture Recognition (FG 2017), pages 612-618, 2017.

[47] Di Wen, Hu Han, and Anil K Jain. Face spoof detection with image distortion analysis. IEEE Transactions on Information Forensics and Security, 10(4):746-761, 2015.

[48] ISO/IEC JTC 1/SC 37 Biometrics. Information technology-Biometric presentation attack detection - Part 1: Framework. International Organization for Standardization, 2016.

[49] G. Huang, Z. Liu, L. Van Der Maaten, and K. Weinberger. Densely connected convolutional networks. In 2017 IEEE Conference on 
Computer Vision and Pattern Recognition (CVPR), pages 4700-4708, 2017.

[50] K. Zhang, Z. Zhang, Z. Li, and Y. Qiao. Joint face detection and alignment using multitask cascaded convolutional networks. IEEE Signal Processing Letters, 23(10):1499-1503, 2016.

[51] D. Kingma and J. Ba. Adam: A method for stochastic optimization. arXiv preprint arXiv:1412.6980, 2014.

[52] H. Chen, Y. Chen, X. Tian, and R. Jiang. A cascade face spoofing detector based on face anti-spoofing r-cnn and improved retinex lbp. IEEE Access, 7:170116-170133, 2019.

[53] F. Zhou, C. Gao, F. Chen, C. Li, X. Li, F. Yang, and Y. Zhao. Face anti-spoofing based on multi-layer domain adaptation. In 2019 IEEE International Conference on Multimedia Expo Workshops (ICMEW), pages 192-197, 2019.

[54] H. Li, P. He, S. Wang, A. Rocha, X. Jiang, and A. C. Kot. Learning generalized deep feature representation for face anti-spoofing. IEEE Transactions on Information Forensics and Security, 13(10):2639-2652, 2018.

[55] H. Chen, G. Hu, Z. Lei, Y. Chen, N. M. Robertson, and S. Z. Li. Attention-based two-stream convolutional networks for face spoofing detection. IEEE Transactions on Information Forensics and Security, 15:578-593, 2020.

[56] J. Galbally and S. Marcel. Face anti-spoofing based on general image quality assessment. In 2014 22nd International Conference on Pattern Recognition (ICPR), pages 1173-1178, 2014.

[57] Z. Boulkenafet, J. Komulainen, Z. Akhtar, A. Benlamoudi, D. Samai, S. E. Bekhouche, A. Ouafi, F. Dornaika, A. Taleb-Ahmed, L. Qin, F. Peng, L. B. Zhang, M. Long, S. Bhilare, V. Kanhangad, A. CostaPazo, E. Vazquez-Fernandez, D. Perez-Cabo, J. J. Moreira-Perez, D. Gonzalez-Jimenez, A. Mohammadi, S. Bhattacharjee, S. Marcel, S. Volkova, Y. Tang, N. Abe, L. Li, X. Feng, Z. Xia, X. Jiang, S. Liu, R. Shao, P. C. Yuen, W. R. Almeida, F. Andalo, R. Padilha, G. Bertocco, W. Dias, J. Wainer, R. Torres, A. Rocha, M. A. Angeloni, G. Folego, A. Godoy, and A. Hadid. A competition on generalized software-based face presentation attack detection in mobile scenarios. In 2017 IEEE International Joint Conference on Biometrics (IJCB), pages 688-696, 2017.

[58] Amin Jourabloo, Yaojie Liu, and Xiaoming Liu. Face de-spoofing: Antispoofing via noise modeling. In Proceedings of the European Conference on Computer Vision (ECCV), pages 290-306, 2018.

[59] X. Yang, W. Luo, L. Bao, Y. Gao, D. Gong, S. Zheng, Z. Li, and W. Liu. Face anti-spoofing: Model matters, so does data. In 2019 IEEE Conference on Computer Vision and Pattern Recognition (CVPR), pages 3502-3511, 2019.

[60] Yaojie Liu, Joel Stehouwer, and Xiaoming Liu. On disentangling spoof trace for generic face anti-spoofing. arXiv preprint arXiv:2007.09273, 2020.

[61] Zezheng Wang, Zitong Yu, Chenxu Zhao, Xiangyu Zhu, Yunxiao Qin, Qiusheng Zhou, Feng Zhou, and Zhen Lei. Deep spatial gradient and temporal depth learning for face anti-spoofing. In Proceedings of the IEEE/CVF Conference on Computer Vision and Pattern Recognition, pages 5042-5051, 2020.

[62] Rui Shao, Xiangyuan Lan, Jiawei Li, and Pong C Yuen. Multiadversarial discriminative deep domain generalization for face presentation attack detection. In Proceedings of the IEEE Conference on Computer Vision and Pattern Recognition, pages 10023-10031, 2019.

[63] Guoqing Wang, Hu Han, Shiguang Shan, and Xilin Chen. Unsupervised adversarial domain adaptation for cross-domain face presentation attack detection. IEEE Transactions on Information Forensics and Security, 16:56-69, 2020.

[64] D. Menotti, G. Chiachia, A. Pinto, W. R. Schwartz, H. Pedrini, A. X. Falcão, and A. Rocha. Deep representations for iris, face, and fingerprint spoofing detection. IEEE Transactions on Information Forensics and Security, 10(4):864-879, 2015.

[65] X. Tu, J. Zhao, M. Xie, G. Du, H. Zhang, J. Li, Z. Ma, and J. Feng. Learning generalizable and identity-discriminative representations for face anti-spoofing. arXiv preprint arXiv:1901.05602, 2019.

[66] R. R. Selvaraju, M. Cogswell, A. Das, R. Vedantam, D. Parikh, and D. Batra. Grad-cam: Visual explanations from deep networks via gradient-based localization. In 2017 IEEE International Conference on Computer Vision (ICCV), pages 618-626, 2017.

[67] J. Hernandez-Ortega, J. Fierrez, A. Morales, and P. Tome. Time analysis of pulse-based face anti-spoofing in visible and nir. In 2018 IEEE/CVF Conference on Computer Vision and Pattern Recognition Workshops (CVPRW), pages 657-6578, 2018.

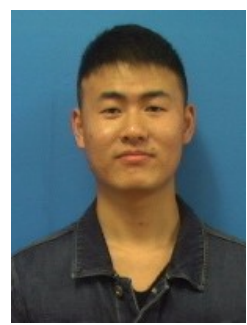

Yahang Wang is a MSc student in Pattern Recognition and Intelligence System in Jiangnan University, Wuxi, China. His current research interests include pattern recognition, computer vision and action recognition.

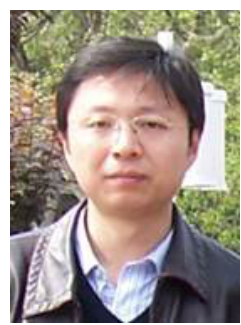

Xiaoning Song received the B.S. degree in computer science from Southeast University, Nanjing, China, in 1997, the M.S. degree in computer science from the Jiangsu University of Science and Technology, Zhenjiang, China, in 2005, and the Ph.D. degree in pattern recognition and intelligence system from the Nanjing University of Science and Technology, Nanjing, in 2010. He was a Visiting Researcher with the Centre for Vision, Speech, and Signal Processing, University of Surrey, Guildford, U.K., from 2014 to 2015. He is currently a Full Professor with the School of Artificial Intelligence and Computer Science, Jiangnan University, Wuxi, China. His current research interests include pattern recognition, machine learning, and computer vision.

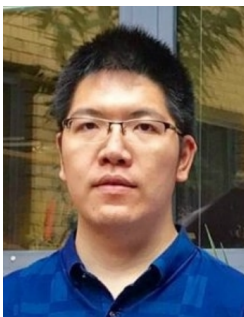

Tianyang Xu received the B.Sc. degree in electronic science and engineering from Nanjing University, Nanjing, China, in 2011. He received the $\mathrm{PhD}$ degree at the School of Artificial Intelligence and Computer Science, Jiangnan University, Wuxi, China, in 2019. $\mathrm{He}$ is currently a research fellow at the Centre for Vision, Speech and Signal Processing (CVSSP), University of Surrey, Guildford, United Kingdom. His research interests include visual tracking and deep learning. He has published several scientific papers, including International Conference on Computer Vision, IEEE Transactions on Image Processing, IEEE Transactions on Circuits and Systems for Video Technology, Pattern Recognition etc. He achieved top 1 tracking performance in the VOT2018 SOT public dataset (ECCV2018) and the Anti-UAV Challenge (CVPR2020).

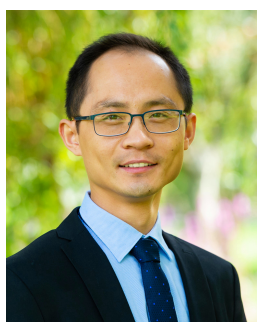

Zhenhua Feng (S'13-M'16) received the Ph.D. degree from the Centre for Vision, Speech and Signal Processing (CVSSP), University of Surrey, Guildford, U.K. in 2016. He is currently a Lecturer in Computer Vision and Machine Learning at the Department of Computer Science, University of Surrey. Before this, he was a senior research fellow at CVSSP. His research interests include pattern recognition, machine learning and computer vision. He has published more than 60 original research papers in top-tier conferences and journals such as TPAMI, IJCV, CVPR, ICCV, IJCAI, ACL, TCYB, TIP, TIFS, TCSVT, TBIOM, ACM TOMM, Information Sciences, Pattern Recognition, etc. He has received the 2017 European Biometrics Industry Award from the European Association for Biometrics (EAB) and the Best Paper Award for Commercial Applications from the AMDO2018 conference. 


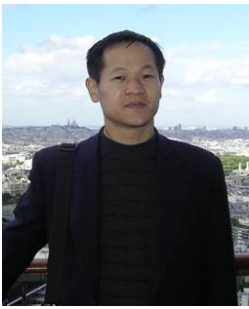

Xiaojun Wu received the B.Sc. degree in mathematics from Nanjing Normal University, Nanjing, China, in 1991, and the M.S. and Ph.D. degrees in pattern recognition and intelligent systems from the Nanjing University of Science and Technology, Nanjing, in 1996 and 2002, respectively. He is currently a Distinguished Professor of Artificial Intelligence and Pattern Recognition with Jiangnan University, Wuxi, China. His current research interests include pattern recognition, computer vision, fuzzy systems, neural networks, and intelligent systems. 